Journal of Mathematics and Statistics 5 (4): 348-351, 2009

ISSN 1549-3644

(C) 2009 Science Publications

\title{
On Numerical Ranges of Nilpotent Elements of C*-Algebra
}

\author{
A. Abdollahi, M.T. Heydari and M. Moosavi \\ Department of Mathematics, Shiraz University, Shiraz 71454, Iran
}

\begin{abstract}
Problem statement: Let A be a $C^{*}$-algebra with unit 1 . For each $a \in A$, let V(a), v(a) and $v_{0}$ (a) denote its numerical range, numerical radius and the distance from the origin to the boundary of its numerical range, respectively. Approach: If a is a nilpotent element of A with the power of nilpotency n, i.e., $a^{n}=0$, and $v(a)=(n-1) v_{0}(a)$. Results: We proved that $V(a)=b W\left(A_{n}\right)$, where $b$ is a scalar and $A_{n}$ is the strictly upper triangular n-by-n matrix with all entries above the main diagonal equal to one. Conclusion/Recommendations: We also completely determined the numerical range of such elements, by determining the numerical range of $\mathrm{W}\left(\mathrm{A}_{\mathrm{n}}\right)$ and showed that the boundary of it does not contain any arc of circle.
\end{abstract}

Key words: Numerical range, numerical radius, $\mathrm{C}^{*}$-algebra, nilpotent

\section{INTRODUCTION}

Let $\mathrm{A}$ be a $\mathrm{C}^{*}$-algebra with unit 1 and let $\mathrm{S}$ be the state space of $A$, i.e., $S=\left\{\varphi \in A^{*}: \varphi \geq 0, \varphi(1)=1\right\}$. For each $a \in A$, the $C^{*}$-algebra numerical range $V(a)$ and numerical radius $v(a)$ is defined, respectively, by:

$$
\mathrm{V}(\mathrm{a}):=\{\varphi(\mathrm{a}): \varphi \in \mathrm{S}\} \text { and } \nu(\mathrm{a}):=\{|\mathrm{z}|: \mathrm{z} \in \mathrm{V}(\mathrm{a})\}
$$

It is well known that $\mathrm{V}(\mathrm{a})$ is non empty, compact and convex subset of the complex plane, $\mathrm{V}(\alpha 1+\beta \mathrm{a})=\alpha+\beta \mathrm{V}(\mathrm{a})$ for $\mathrm{a} \in \mathrm{A}$ and $\alpha, \beta \in \mathbb{C}$ and if $\mathrm{z} \in \mathrm{V}(\mathrm{a}),|\mathrm{z}| \leq\|\mathrm{a}\|^{[2]}$.

The notion of numerical range or the classical field of values was first introduced by Toeplitz in 1918 for matrices. This concept were independently extended by G. Lumer and F. Bauer in sixties to a bounded linear operator on an arbitrary Banach space. In 1975, Lightbourne and Martin ${ }^{[9]}$ have extended this concept by employing a class of seminorms generated by a family of supplementary projections.

As an example, let $\mathrm{A}$ be the $\mathrm{C}^{*}$-algebra of all bounded linear operators on a complex Hilbert space $\mathrm{H}$ and $\mathrm{T} \in \mathrm{A}$. It is well known that $\mathrm{V}(\mathrm{T})$ is the closure of $\mathrm{W}(\mathrm{T})$, where:

$$
\mathrm{W}(\mathrm{T}):=\{\langle\mathrm{Tx}, \mathrm{x}\rangle: \mathrm{x} \in \mathrm{H},\|\mathrm{x}\|=1\}
$$

is the usual numerical range of the operator $\mathrm{T}$. In this special case we denote the numerical radius of $\mathrm{T}$ and the distance from the origin to the boundary of its numerical range by $\mathrm{w}(\mathrm{T})$ and $\mathrm{w}_{0}(\mathrm{~T})$, respectively.
A complete survey on numerical range can be found in the books by Bonsall and Duncan ${ }^{[2,3]}$ and the book by Gustafon and $\mathrm{Rao}^{[5]}$ and we refer the reader to these books for general information and background.

In 1992, Haagerup and de la Harpe ${ }^{[6]}$ have proved the following sharp estimate for the numerical radius of a nilpotent operator $\mathrm{N}$ :

$$
\mathrm{w}(\mathrm{N}) \leq\|\mathrm{N}\| \cos \left(\frac{\pi}{\mathrm{n}+1}\right)
$$

where, $n$ is the power nilpotency of $n$. In 2004, Karaev give another proofs of the Haagerup-de la Harpe inequality.

In [4] the researchers have shown that if $\mathrm{A}$ is a nonzero nilpotent operator with the power of nilpotency $\mathrm{n}$, with $\mathrm{w}(\mathrm{A}) \leq(\mathrm{n}-1) \mathrm{w}_{0}(\mathrm{~A})$ and if $\mathrm{A}$ attains its numerical radius then the following conditions are equivalent:

- $\quad \mathrm{w}(\mathrm{A})=(\mathrm{n}-1) \mathrm{w}_{0}(\mathrm{~A})$

- $\mathrm{A}$ is unitarily equivalent to an operator of the form $\eta A_{n} \oplus A^{\prime}$, where $\eta$ is a scalar satisfying $|\eta|=2 \mathrm{w}_{0}(A)$ and $\mathrm{A}^{\prime}$ is some other operator:

- $\quad \mathrm{W}(\mathrm{A})=\mathrm{bW}\left(\mathrm{A}_{\mathrm{n}}\right)$

Where:

$$
\mathrm{b}=\mathrm{A} \text { scalar }
$$

$$
\mathrm{A}_{\mathrm{n}}:=\left[\begin{array}{cccc}
0 & 1 & \cdots & 1 \\
& 0 & \ddots & \vdots \\
& & \ddots & 1 \\
& & & 0
\end{array}\right]
$$




\section{MATERIALS AND METHODS}

Let a be a nilpotent with the power of nilpotency $n \geq 1$, i.e., $a^{n}=0$ and $v(a)=(n-1) v_{0}(a)$, where $v_{0}(a)$ denotes the distance from the origin to the boundary of its numerical range. In this study we show that $\mathrm{V}(\mathrm{a})=\mathrm{bW}\left(\mathrm{A}_{\mathrm{n}}\right)$, where $\mathrm{b}$ is a scalar and by determining the boundary of numerical range of $A_{n}$, we show that the $\partial \mathrm{V}$ (a) not inclusive a circle section. Actually, this study is an extension of the Haagerup-de and la Harpe inequality and the research of an earlier study by $\mathrm{Gau}^{[4]}$ to the $\mathrm{C}^{*}$-algebra numerical range.

A short survey on $\mathbf{W}\left(\mathbf{A}_{\mathbf{n}}\right)$ : For the study of numerical ranges of finite matrices, the matrix-theoretic properties can be exploited to yield special tools which are not available for general operators. One important way to yield $\partial \mathrm{W}(\mathrm{A})$ is the Kippenhahn's result that the numerical range of A coincides with the convex hull of the real points of the dual curve of $\operatorname{det}(\mathrm{xReA}+\mathrm{ylmA}+$ $\mathrm{zl})=0^{[4]}$. On the other hand, a parametric representation of the boundary W(A) can also be obtained from the largest eigenvalue of $\operatorname{Re}\left(\mathrm{e}^{-\mathrm{i} \theta} \mathrm{A}\right)$ yielding useful information on $\mathrm{W}(\mathrm{A})$.

For any $n \times n$ matrix $A$, let $\lambda(\theta)$ denote the maximum eigenvalue of $\operatorname{Re}\left(\mathrm{e}^{-\mathrm{i} \theta} \mathrm{A}\right)$. It is well known that $\lambda(\theta)$ is an analytic function of $\theta$ (possibly except for some isolated points) and a unit vector in $\mathbb{C}^{\mathrm{n}}$ is such that $\langle A x, x\rangle$ belong to $\partial W(A) \cap L_{\theta}$ if and only if $\operatorname{Re}\left(\mathrm{e}^{-\mathrm{i} \theta} \mathrm{A}\right) \mathrm{x}=\lambda(\theta) \mathrm{x}^{[4]}$. Also $\partial \mathrm{W}(\mathrm{A})$ admits a parametric representation:

$\mathrm{x}(\theta)=\lambda(\theta) \cos (\theta)-\lambda^{\prime}(\theta) \sin (\theta)$

$y(\theta)=\lambda(\theta) \sin (\theta)+\lambda^{\prime}(\theta) \cos (\theta)$

(again, with possible exception of finitely many points). The curvature and radius of curvature of $\partial \mathrm{W}(\mathrm{A})$ at $\mathrm{p}=$ $(\mathrm{x}(\theta), \mathrm{y}(\theta))$ are equals:

$$
\mathrm{K}(\theta)=\frac{1}{\lambda(\theta)+\lambda^{\prime \prime}(\theta)}
$$

and

$$
\mathrm{R}(\theta)=\lambda(\theta)+\lambda^{\prime \prime}(\theta),
$$

respectively.

As we mentioned, if $\mathrm{A}$ is a nilpotent operator on a Hilbert space $\mathrm{H}$ with nilpotency $\mathrm{n}$ that attaint its numerical radius and $\mathrm{w}(\mathrm{A})=(\mathrm{n}-1) \mathrm{w}_{0}(\mathrm{~A})$ then $\mathrm{W}(\mathrm{A})=\mathrm{bW}\left(\mathrm{A}_{\mathrm{n}}\right)$, for some $\mathrm{b}$. Hence for determining $W(A)$ it is enough to compute $W\left(A_{n}\right)$. The following theorem can be help us to find $\mathrm{W}(\mathrm{A})$.

\section{RESULTS AND DISCUSSION}

Theorem 1: The boundary of $\mathrm{W}\left(\mathrm{A}_{\mathrm{n}}\right)$ is:

$$
\partial \mathrm{W}\left(\mathrm{A}_{\mathrm{n}}\right)=\gamma_{1}+\gamma_{2}
$$

Where:

$$
\gamma_{2}(t)=-\frac{1}{2}+i\left(\frac{-3}{2} \cot \left(\frac{\pi}{n}\right) t+\frac{1}{2} \cot \left(\frac{\pi}{n}\right)\right)
$$

for $0 \leq \mathrm{t} \leq 1$ and:

$$
\gamma_{1}(\theta)=\left(\lambda(\theta)+i \lambda^{\prime}(\theta)\right) \mathrm{e}^{\mathrm{i} \theta}
$$

which $\lambda(0)=\frac{\mathrm{n}-1}{2}$ and for $-\pi \leq \theta \leq \pi, \theta \neq 0$ :

$$
\lambda(\theta)=\frac{1}{2}\left[\sin (\theta) \cot \left(\frac{\theta}{n}\right)-\cos (\theta)\right]
$$

$\partial \mathrm{W}\left(\mathrm{A}_{\mathrm{n}}\right)$ is differentiable at each points.

The curvature function of the boundary of numerical range of $A_{n}, \partial W\left(A_{n}\right)$, is:

$$
\mathrm{R}(\theta)=\frac{1}{\mathrm{n}^{2}} \csc ^{3}\left(\frac{\theta}{\mathrm{n}}\right)-\left[\sin (\theta) \cos \left(\frac{\theta}{\mathrm{n}}\right)-\mathrm{n} \cos (\theta) \sin \left(\frac{\theta}{\mathrm{n}}\right)\right]
$$

Proof: First we want to compute the $\lambda(\theta)$. Put $M_{n}=$ $\operatorname{Re}\left(e^{i \theta} A_{n}\right)-\lambda I_{n}$ and $P_{n}(\lambda)=\operatorname{det} M_{n}$. Therefore:

$$
P_{n}(\lambda)=\operatorname{det}\left[\begin{array}{ccccc}
-\lambda & \frac{\mathrm{e}^{-i \theta}}{2} & \frac{\mathrm{e}^{-i \theta}}{2} & \cdots & \frac{\mathrm{e}^{-i \theta}}{2} \\
\frac{\mathrm{e}^{\mathrm{i} \theta}}{2} & -\lambda & \frac{\mathrm{e}^{-i \theta}}{2} & \cdots & \frac{\mathrm{e}^{-i \theta}}{2} \\
\frac{\mathrm{e}^{\mathrm{i} \theta}}{2} & \frac{\mathrm{e}^{\mathrm{i} \theta}}{2} & -\lambda & \cdots & \frac{\mathrm{e}^{-i \theta}}{2} \\
\vdots & \vdots & \vdots & \ddots & \vdots \\
\frac{\mathrm{e}^{\mathrm{i} \theta}}{2} & \frac{\mathrm{e}^{\mathrm{i} \theta}}{2} & \frac{\mathrm{e}^{\mathrm{i} \theta}}{2} & \cdots & -\lambda
\end{array}\right]
$$

by adding the -1 multiple of any row to before row we have:

$$
\mathrm{P}_{\mathrm{n}}(\lambda)=\operatorname{det}\left[\begin{array}{ccccc}
-\lambda-\frac{\mathrm{e}^{\mathrm{i} \theta}}{2} & \lambda+\frac{\mathrm{e}^{-\mathrm{i} \theta}}{2} & 0 & \cdots & 0 \\
0 & -\lambda-\frac{\mathrm{e}^{\mathrm{i} \theta}}{2} & \lambda+\frac{\mathrm{e}^{-\mathrm{i} \theta}}{2} & \cdots & 0 \\
0 & 0 & -\lambda-\frac{\mathrm{e}^{-\mathrm{i} \theta}}{2} & \cdots & 0 \\
\vdots & \vdots & \vdots & \ddots & \vdots \\
\frac{\mathrm{e}^{\mathrm{i} \theta}}{2} & \frac{\mathrm{e}^{\mathrm{i} \theta}}{2} & \frac{\mathrm{e}^{\mathrm{i} \theta}}{2} & \cdots & -\lambda
\end{array}\right]
$$


Important, but easy to check, is the fact that when expanding this determinant about the first column, we have the following Recursion formula:

$$
P_{n}(\lambda)=\left(-\lambda-\frac{e^{i \theta}}{2}\right) P_{n-1}(\lambda)+(-1)^{n-1} \frac{e^{i \theta}}{2}\left(\lambda+\frac{e^{-i \theta}}{2}\right)^{n-1}, n \geq 1
$$

with initial condition $\mathrm{P}_{\mathrm{o}}(\lambda)=1$.

By solving this recursion formula we have:

$$
P_{n}(\lambda)=\frac{(-1)^{n+1}}{e^{i \theta}-e^{-i \theta}}\left[e^{-i \theta}\left(\lambda+\frac{e^{i \theta}}{2}\right)^{n}-e^{i \theta}\left(\lambda+\frac{e^{-i \theta}}{2}\right) n\right]
$$

if $\theta \neq m \pi$, for each $m \in \mathbb{Z}$ and:

$$
\mathrm{P}_{\mathrm{n}}(\lambda)=\frac{(-1)^{\mathrm{n}+1}}{2}(\mathrm{n}-2 \lambda-1)\left(\lambda+\frac{1}{2}\right)^{\mathrm{n}-1}
$$

whenever $\theta=m \pi$, for some $m \in \mathbb{Z}$.

By solving $\mathrm{P}_{\mathrm{n}}(\lambda)=0$, in the first case we have:

$$
\left(\frac{\lambda+\frac{\mathrm{e}^{\mathrm{i} \theta}}{2}}{\lambda+\frac{\mathrm{e}^{-\mathrm{i} \theta}}{2}}\right)^{\mathrm{n}}=\mathrm{e}^{2 \mathrm{i} \theta}
$$

Which implies:

$$
\lambda_{\mathrm{k}}=\frac{1}{2}\left[\sin \theta \cot \left(\frac{\mathrm{k} \pi+\theta}{\mathrm{n}}\right)-\cos \theta\right], \mathrm{k}=0,1, \ldots, \mathrm{n}-1
$$

By a simple computation we see that for $\theta \neq 0$, $\lambda(\theta)=\lambda_{0} \geq \lambda_{\mathrm{k}}$, for each $\mathrm{k}$ and so $\lambda(\theta)$ satisfies (a). This relation with Eq. 1 and inequality $\operatorname{sink} \theta \leq \mathrm{k} \sin \theta$ implies that $\mathrm{w}\left(\mathrm{A}_{\mathrm{n}}\right)=\frac{\mathrm{n}-1}{2}$ and $\mathrm{w}_{0}\left(\mathrm{~A}_{\mathrm{n}}\right)=\frac{1}{2}$, independent from $\mathrm{n}$.

This facts will help in discussing and proving many of the result below. Therefore let $-\pi<\theta<\pi$ and put:

$$
\gamma_{1}(\theta)=\left(\lambda(\theta)+\mathrm{i} \lambda^{\prime}(\theta)\right) \mathrm{e}^{\mathrm{i} \theta}
$$

Also define:

$$
\gamma_{2}(t)=-\frac{1}{2}+i\left(\frac{-3}{2} \cot \left(\frac{\pi}{n}\right) t+\frac{1}{2} \cot \left(\frac{\pi}{n}\right)\right)
$$

for $0 \leq t \leq 1$. So we have:

$$
\partial \mathrm{W}\left(\mathrm{A}_{\mathrm{n}}\right)=\gamma_{1}+\gamma_{2}
$$

It is easy to show that $\partial \mathrm{W}\left(\mathrm{A}_{\mathrm{n}}\right)$ is differentiable.
By direct calculation we have:

$$
\lambda^{\prime}(\theta)=\frac{1}{2}\left[\cos (\theta) \cot \left(\frac{\theta}{n}\right)-\frac{1}{n} \sin (\theta) \csc ^{2}\left(\frac{\theta}{n}\right)+\sin (\theta)\right]
$$

for $-\pi \leq \theta \leq \pi, \theta \neq 0$ and $\lambda^{\prime}(0)=0$ also:

$$
\lambda^{\prime \prime}(\theta)=\frac{1}{2}\left[\begin{array}{c}
-\sin (\theta) \cot \left(\frac{\theta}{\mathrm{n}}\right)-\frac{2}{\mathrm{n}} \cos (\theta) \csc ^{2}\left(\frac{\theta}{\mathrm{n}}\right) \\
+\frac{1}{\mathrm{n}^{2}} \sin (\theta) \cos \left(\frac{\theta}{\mathrm{n}}\right) \csc ^{3}\left(\frac{\theta}{\mathrm{n}}\right) \cos (\theta)
\end{array}\right]
$$

for $-\pi \leq \theta \leq \pi, \theta \neq 0$ and $\lambda^{\prime \prime}(0)=0$ So the curvature function of the boundary of numerical range of $A_{n}$, $\partial \mathrm{W}\left(\mathrm{A}_{\mathrm{n}}\right)$, is:

$$
\mathrm{R}(\theta)=\frac{1}{\mathrm{n}^{2}} \csc ^{3}\left(\frac{\theta}{\mathrm{n}}\right)\left[\sin (\theta) \cos \left(\frac{\theta}{\mathrm{n}}\right)-\mathrm{n} \cos (\theta) \sin \left(\frac{\theta}{\mathrm{n}}\right)\right]
$$

Now the proof is completed.

This proof will help in discussing the following corollary:

Corollary 1: By radius of curvature, $\partial \mathrm{W}\left(\mathrm{A}_{\mathrm{n}}\right)$ does not contain any arc of circle.

$C^{*}$-algebra numerical range of nilpotent elements: As we mentioned in the introduction, let $\mathrm{A}$ be a $\mathrm{C}^{*}$ algebra with unit 1 and let $S$ be the state space of $A$, i.e., $S=\left\{\varphi \in A^{*}: \varphi \geq 0, \varphi(1)=1\right\}$. For each $a \in A$, the $C^{*}$ algebra numerical range is defined by:

$$
\mathrm{V}(\mathrm{a}):=\{\varphi(\mathrm{a}): \varphi \in \mathrm{S}\}
$$

Analogy we define $v(a)$ and $v_{0}(a)$ for an element $a \in A$ by:

$$
v(a)=\sup \{|z|: z \in \partial V(a)\}
$$

and

$$
\mathrm{v}_{0}(\mathrm{a})=\operatorname{dist}(0, \partial \mathrm{V}(\mathrm{a}))=\inf \{|\mathrm{z}|: \mathrm{z} \in \partial \mathrm{V}(\mathrm{a})\}
$$

Now we turn our attention to nilpotency.

Theorem 2: Let $\mathrm{A}$ be a $\mathrm{C}^{*}$-algebra with unit and $\mathrm{a} \in \mathrm{A}$ be a nilpotent element with nilpotency $\mathrm{n}$. Then:

$$
v(a) \leq\|a\| \cos \left(\frac{\pi}{n+1}\right)
$$


If $v(a)=(n-1) v_{0}(a)$, then:

$$
\mathrm{V}(\mathrm{a})=\mathrm{bW}\left(\mathrm{A}_{\mathrm{n}}\right)
$$

where, $\mathrm{b}$ is a scalar.

$$
\|\mathrm{a}\| \geq|\mathrm{b}| \frac{\mathrm{n}-1}{2} \sec \frac{\pi}{\mathrm{n}+1}
$$

$\partial \mathrm{V}(\mathrm{a})$ does not contain any arc of circle.

Proof: Let $\rho$ be a state of A. Then there exists a cyclic representation $\pi_{\rho}$ of $\mathrm{A}$ on a Hilbert space $\mathrm{H}_{\rho}$ and a unit cyclic vector $\mathrm{x}_{\rho}$ for $\mathrm{H}_{\rho}$ such that:

$$
\rho(a)=\left\langle\pi_{\rho}(a) x_{\rho}, x_{\rho}\right\rangle, a \in A
$$

By Gelfand-Naimark theorem the direct sum $\pi: \mathrm{a} \rightarrow \sum_{\rho \in \mathrm{s}} \oplus \pi_{\rho}(\mathrm{a})$ is a faithful representation of $\mathrm{A}$ on the Hilbert space $\mathrm{H}=\sum_{\rho \in \mathrm{s}} \oplus \mathrm{H}_{\rho}{ }^{[8]}$. Therefore for each $\rho \in S, \quad \rho(a) \in W\left(\pi_{\rho}\right)(a) \subset W(\pi(a)) \quad$ and hence $V(a)$ contained in $\mathrm{W}(\pi(\mathrm{a}))$. On the other hand if $\mathrm{x}$ is a unit vector of $H$, then the formula $\rho(\mathrm{c})=\langle\pi(\mathrm{c}) \mathrm{x}, \mathrm{x}\rangle, \mathrm{c} \in \mathrm{A}$, defines a state on $A$ and hence $\rho(a)=\langle\pi(a) x, x\rangle \in V(a)$ and it follows that:

$$
\mathrm{W}\left(\mathrm{T}_{\mathrm{a}}\right)=\mathrm{V}(\mathrm{a})
$$

where, $\mathrm{T}_{\mathrm{a}}=\pi(\mathrm{a})^{[1]}$.

Also $\mathrm{a}^{\mathrm{n}}=0$ implies that $\mathrm{T}_{\mathrm{a}}^{\mathrm{n}}=0, \mathrm{~T}_{\mathrm{a}}$ is a nilpotent operator with nilpotency $n$. Then (i) follows from (1) and (2). Part (iv) follows from Theorem 1. Also $w\left(T_{a}\right)=(n-1)$ $\mathrm{w}_{0}\left(\mathrm{~T}_{\mathrm{a}}\right)$ and so $\mathrm{V}(\mathrm{a})=\mathrm{W}\left(\mathrm{T}_{\mathrm{a}}\right)=\mathrm{bW}\left(\mathrm{A}_{\mathrm{n}}\right)$, where $\mathrm{b}$ is a scalar, which implies (ii). Finally (iii) follows from (i) and the facts that $v(a)=|b| w\left(A_{n}\right)$ and $w\left(A_{n}\right)=\frac{n-1}{2}$.

\section{CONCLUSION}

If $\mathrm{A}$ is a $\mathrm{C}^{*}$-algebra with unit and $\mathrm{a} \in \mathrm{A}$ is a nilpotent element with nilpotency $n$ and $v(a)=(n-$ 1) $v_{0}(a)$, then:

$$
\mathrm{V}(\mathrm{a})=\mathrm{bW}\left(\mathrm{A}_{\mathrm{n}}\right)
$$

where, $b$ is a scalar. Also $\partial \mathrm{V}(\mathrm{a})$ does not contain any arc of circle.

So, we can completely determine the numerical range of such elements.

\section{REFERENCES}

1. Berberian, S.K. and G.H. Orland, 1967. On the closure of the numerical range of an operator. Proc. Am. Math. Soc., 18: 499-503. http://www.jstor.org/stable/2035486

2. Bonsall, F.F., J. Duncan and N.J. Hitchin, 1971. Numerical Ranges of Operators on Normed Spaces and of Elements of Normed Algebras, Cambridge University Press, Cambridge, ISBN: 13: 9780521079884, pp: 148.

3. Bonsall, F.F. and J. Duncan, 1973. Numerical Ranges II. Cambridge University Press, Cambridge, ISBN: 10: 052120227, pp: 188.

4. Gau, H.L. and P.Y. Wu, 2008. Numerical ranges of nilpotent operators. Linear Algebra Applied, 429: 716-726.

5. Gustafon, K.E. and K.M. Rao, 1996. The Numerical Range: The Field of Values of Linear Operators and Matrices. 1st Edn., Springer, New York, ISBN: 10: 038794835X, pp: 189.

6. Haagerup, U. and P. de la Harpe, 1992. The numerical radius of a nilpotent operator on a Hilbert space. Proc. Am. Math. Soc., 115: 371-379. http://www.jstor.org/stable/2159255

7. Halmos, P.R., 1982. A Hilbert Space Problem Book. 2nd Edn., Springer, New York, ISBN: 3540-90685-1.

8. Kadison, R.V. and J.R. Ringrose, 1983. Fundamentals of the Theory of Operator Algebras: Elementary Theory. Academic Press, New York, ISBN: 10: 0123933013, pp: 416.

9. Lightbourne, J.H. and R.H. Martin, 1975. Projection seminorms and the field of values of linear operators. Num. Math., 24: 151-161. DOI: 10.1007/BF01400964 